\title{
Single-cell transcriptomics: a novel precision medicine technique in nephrology
}

Jisoo Kim and Jihwan Park

School of Life Sciences, Gwangju Institute of Science and Technology, Gwangju, Korea

\section{Received: August 11, 2020 \\ Accepted: October 21, 2020}

\section{Correspondence to}

Jihwan Park, Ph.D.

School of Life Sciences, Gwangju Institute of Science and Technology, 123 Cheomdan-gwagiro, Buk-gu, Gwangju 61005, Korea

Tel: +82-62-715-2503

Fax: $+82-62-715-2484$

E-mail:jihwan.park@gist.ac.kr https://orcid.org/0000-0002$5728-912 \mathrm{X}$
Due to the complex structure and function of the kidneys, the mechanism of kidney disease is unclear. In particular, transcriptomics approaches at the bulk level are unable to differentiate primary autonomous responses, which lead to disease development, from secondary cell non-autonomous responses. Single-cell analysis techniques can overcome the limitations inherent in the measurement of heterogeneous cell populations and clarify the central issues in kidney biology and disease pathogenesis. Single-cell sequencing helps in identifying disease-related biomarkers and pathways, stratifying patients, and deciding on appropriate treatment methods. Here we review a variety of single-cell analysis techniques and single-cell transcriptomics studies performed in the field of nephrology. Moreover, we discuss the future prospects of single-cell analysis-based precision medicine in nephrology.

Keywords: Precision medicine; Single-cell; Nephrology

\section{INTRODUCTION}

Defining the regulatory mechanisms that determine cell function and behavior is fundamental for understanding disease. However, due to the complex structure and function of the kidneys, the mechanisms governing the development and progression of kidney disease are unclear. Although RNA sequencing has enhanced our understanding of the causes and progress of kidney diseases, the bulk transcriptome does not allow analysis of biological differences between cell types. To overcome this, single-cell RNA sequencing (scRNA-seq) was developed. scRNA-seq involves single-cell isolation, transcript capture, library construction, sequencing, and computational analysis, and enables evaluation of the biological properties of individual cells and cell populations at high resolution. It is a powerful approach to analyzing not only the complex processes of kidney diseases but also the underlying causal mechanisms, facilitating identification of therapeutic targets.
Precision medicine characterizes patients based on information on all factors that can affect treatment, from dysregulation of genes and cells to lifestyle, diet, and environment. Precision medicine aims to identify biomarkers of treatment success more rapidly than the standard determination. Second, it identifies pathways for therapeutic intervention using existing pharmacological or novel target specific agents [1]. Identification of specific target genes, signaling pathways, and primary target cell types is needed to enable precision medicine in nephrology. Although much effort has focused on identifying target genes, cellular targets in kidney diseases are unclear because of technical limitations.

Application of scRNA-seq can facilitate precision medicine by defining the cell types involved in complex conditions such as chronic kidney disease (CKD) and acute kidney injury (AKI). Moreover, scRNA-seq can identify potential biomarkers and signaling pathways to enable diagnosis, prognosis evaluation, targeted therapy, early detection, and noninvasive monitoring (Fig. 1). 


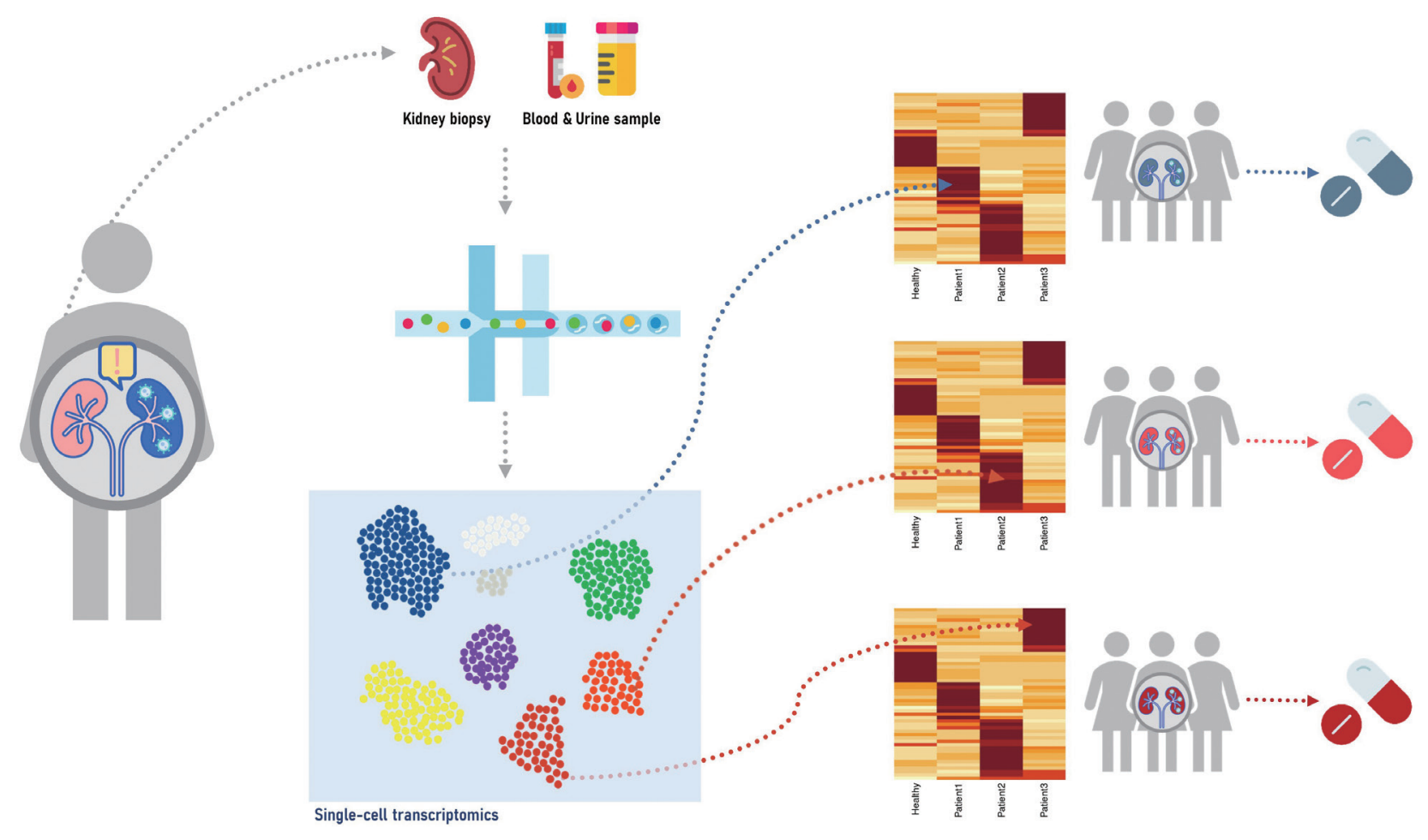

Figure 1. Application of single-cell transcriptomics to precision medicine for kidney diseases.

Therefore, scRNA-seq can realize precision medicine in nephrology. Here we review several single-cell analysis techniques and scRNA-seq studies of the kidneys. Moreover, we discuss the application of single-cell analysis to precision medicine in nephrology.

Single-cell analysis can be performed on kidney biopsies, blood, and urine and has high resolution that allows observation of disease transitions at the single-cell level. Single-cell transcriptomics will assist identification of disease-related biomarkers and pathways. This will enable more accurate classification of patients and administration of tailored treatments.

\section{METHODOLOGICAL OVERVIEW}

\section{Sample preparation for single-cell analysis}

There are several experimental considerations for scRNAseq such as tissue preservation, tissue dissociation, and cell count.

Obtaining high-quality single-cell suspensions is a key determinant of the success of single-cell studies. The single-cell preparation process is the most frequent cause of unwanted technical variation and batch effects in single-cell studies. To obtain a single-cell suspension from kidney samples, fresh tissue is dissociated by mechanical disruption and enzymatic digestion. An automatic tissue dissociator is typically used to minimize batch effects. Because different tissues have different characteristics, the protocol must be optimized for each purpose. Excessive tissue dissociation causes cell damage and reduces viability, which can result in unwanted transcriptional changes, ambient mRNAs, and greater amounts of mitochondrial mRNAs $[2,3]$. In contrast, insufficient tissue dissociation leads to multiplets in the data. In addition, a single-cell suspension obtained by tissue dissociation should be filtered through a cell strainer of appropriate size or immersed in cell debris removal solution. Cell counting is also critical because use of an excessive cell density can result in capture of multiple cells simultaneously. In contrast, underloading can cause loss of information due to empty drop- 
lets. Multiplets can be removed using computational tools such as Scrublet and DoubletFinder $[4,5]$. It is also important to determine the appropriate number of cells to be analyzed, which requires consideration of sample heterogeneity and abundance of the target cell type. A large number of cells must be sequenced to analyze small populations of renal cells $(20,000$ cells are required to obtain 100 target cells if the target cell type frequency is $0.5 \%$ ), as approximately $60 \%$ of the kidney is composed of proximal tubule cells [6].

scRNA-seq analysis is hindered by difficulties in isolating cells or if cells are damaged. Single nucleus RNA sequencing (snRNA-seq) overcomes these limitations by isolating a single nucleus rather than a single-cell $[7,8]$. Although scRNA-seq requires fresh tissue, snRNA-seq can be performed on frozen tissue, and can be applied to tissues that are difficult to separate because of intertwined cells [8]. Furthermore, snRNA-seq reduces cell stress and the composition bias generated during the separation step. Nevertheless, information on cytoplasmic RNA cannot be obtained because only nuclear RNAs are analyzed, and various intron sequences are present. Therefore, the method used should be selected based on the aim of the experiment.

\section{Single RNA sequencing technologies}

scRNA-seq methods typically involve RNA capture, reverse transcription, complementary DNA (cDNA) amplification, sequencing library construction, and high-throughput sequencing.

scRNA-seq is mainly divided into full-length scRNAseq and tag-based scRNA-seq according to library construction method. Full-length scRNA-seq can be utilized not only to measure gene expression levels but also to identify transcript isoforms, alternative splicing, and single-nucleotide polymorphisms within the transcripts [9-12]. It has high sequencing coverage and mapping efficiency; however, it has limited cell throughput (hundreds of cells), a relatively large batch effect, considerable sample preparation time, and high cost per cell because the samples must be prepared independently [9,13-15]. Smart-seq2 and Quartz-seq are representative full-length scRNA-seq methods $[16,17]$. The tag-based scRNA-seq technique estimates transcript abundance by sequencing the $3^{\prime}$-end of transcripts in a large number of cells (tens of thousands to millions of cells) $[9,13,18,19]$. To distinguish cell types and to determine the transcript copy number accurately, cDNA molecules are labeled with barcode sequences such as cell barcodes and unique molecular identifiers (UMIs). Tag-based scRNA-seq is subdivided into droplet-based [19-21], microwell-based [22,23], and split-pool barcoding-based technologies $[24,25]$, according to the labeling method. Droplet-based technology uses a microfluidic device to generate aqueous droplets formed by combining water and oil streams. In each droplet, one cell and one barcoded bead pair are encapsulated, and each single-cell mRNA is captured by oligo-dT of the barcoded bead. During cDNA synthesis, cell barcodes and UMIs are added to the cDNAs [19-21]. Although droplet-based barcoding generates limited information on the $3^{\prime}$-end of the mRNA, it improves the throughput of single-cell analysis and reduces the time, labor, cost per cell, and batch effect by simplifying the experimental procedure. Microwell-based technology captures mRNA by loading cells on a microwell plate, washing doublets with capillaries, and adding barcoded beads [22,23]; it is simple and economical. Split-pool barcode methods identify a single-cell by combinatorial indexing without the need for separation to obtain a single-cell. Single-cell combinatorial indexing RNA sequencing (sci-RNA-seq) and split-pool ligation-based transcriptome sequencing (SPLiT-seq) are typical split-pool barcoding methods $[24,25]$. These techniques distribute permeabilized cells across 96- or 384-well plates; the first molecular index is introduced to the mRNA of cells in each well, with in situ reverse transcription. After the first barcoding, the cells are pooled, and the cells are distributed across another plate. Thereafter, a second barcode is added, resulting in a unique combination of barcodes for each cell $[24,25]$.

Another challenge of scRNA-seq is the loss of cell location and orientation information during tissue dissociation. Much effort has focused on recreating a near-realistic cell environment. Because cells exist in a three-dimensional $\left.{ }_{3} \mathrm{D}\right)$ space and interact within that space, spatial transcriptome analysis was developed to evaluate cell types and their locations. Single-molecule fluorescence in situ hybridization (smFISH) was used to identify RNA location and copy number; however, it cannot analyze multiple cells simultaneously [26,27]. To overcome this limitation, multiplexed error robust FISH (MERFISH), which involves labeling of multiple RNAs 
in a single-cell, and sequential-FISH (seq-FISH), which involves multiple rounds of FISH, have been developed $[28,29]$. In addition, spatially resolved transcript amplicon readout mapping, which labels RNA with a DNA probe, and fluorescent in situ sequencing, which overcomes the shortcomings of existing padlock probes, are available $[30,31]$. Visium, a spatial transcriptomics technology from 10x Genomics, provides a multidimensional view of tissue biology by means of high-throughput mRNA analysis of intact tissue sections [32]. Computing technologies such as Seurat, DistMap, and novoSpaRc are also evolving [33-35], and efforts are focused on understanding the functions of cells in $3 \mathrm{D}$ space.

scRNA-seq has several limitations that need to be overcome. For example, dissociation may cause stress or alter the cell proportion $[2,36]$. Also, the batch effect may result from use of different protocols, sample handling, and platforms $[37,38]$. In addition, the transcript efficiency per cell is lower compared to bulk RNA-seq [39,40]. Finally, dropout occurs with droplet-based scRNA-seq $[20,21,41]$.

\section{Computational analysis of scRNA-seq data}

The raw data generated by sequencing are processed using analysis pipelines such as Cell Ranger [21], SEQC [42], and zUMIs [43] to a gene-by-cell data matrix.

Quality control filters select only high-quality cells, facilitating identification of cell type, and screening of failed samples so that their data can be recovered or removed from analysis. This requires generation of quality metrics such as the number of UMIs per cell, number of genes detected per cell, and proportion of mitochondrial genes [44-46].

Normalization is essential for accurate comparison of gene expression between samples. Because the gene expression count depth of the same cells can vary due to the diversity inherent in each step-such as single-cell capture, reverse transcription, and sequencing-the gene expression counts are scaled by the total number of sequencing reads or counts per cell $[45,47,48]$.

After normalization, clustering analysis is performed to separate the cells based on gene expression patterns and identify the cell types. The Seurat package allocates cells to clusters based on the principal component scores obtained from the expression of the most variable genes [49,50]. For visualization of the cell clusters, t-distributed stochastic neighbor embedding and uniform manifold approximation and projection are typically performed $[51,52]$. The identified clusters are then assigned into known cell types based on cell type-specific markers or by automatic cell assignment software. There are several cell-marker databases for cell type classification, such as Cell Finder [53], CellMarker [54], and PanglaoDB [55]. Despite marker-based cell type classification being commonly used, these methods cannot distinguish other cell types that express the same marker from the target cell type, and can complicate cell type classificaition by the heterogeneity of cell states. The various automated cluster annotation methods combine annotation and clustering, and the automatic cell identification methods are compared elsewhere [56]. Downstream analyses—such as trajectory analysis, differential expression analysis, gene set analysis, and gene regulatory networks-have been reviewed by others $[45,47,57]$.

\section{Integration of single-cell transcriptomics with other -omics data}

Single-cell sequencing enables simultaneous analysis of the genome, epigenome, transcriptome, and proteome of one cell. By integrating genomic and transcriptomic information, it is possible to confirm the effect of DNA copy number variation on gene expression, transcript changes according to genomic changes, and the effect of mutations in the coding or non-coding region on transcript expression [58]. Genome and transcriptome sequencing (G\&T-seq) combines whole-genome amplification and Smart-seq2 [59]. Single-cell methylome and transcriptome (scM and T-seq) was developed to analyze the relationship between DNA methylation and the transcriptome in a single-cell $[60,61]$.

Moreover, technologies that integrate chromatin information and the transcriptome are now available. Single-cell combinatorial indexing-chromatin accessibility and mRNA (sci-CAR) integrates single-cell transcriptome analysis (sci-RNA-seq) and epigenetic analysis (sciATAC-seq) [62]. This allows assessment of the relationships between differentially expressed genes and their regulatory chromatin regions.

RNA and protein determine the properties of a biological system; however, because of the distinct half-lives of mRNA and protein, and the effects of post-transcriptional modification, it is difficult to evaluate the correla- 
tion between mRNA and protein levels [58]. Simultaneous identification of the transcriptome and proteome at the single-cell level has enabled exploration of RNA and protein abundance. Cellular indexing of transcriptomes and epitope sequencing (CITE-seq) and RNA expression and protein sequencing (REAP-seq) enables simultaneous analysis of proteins and transcripts at the single-cell level using oligonucleotide-labeled antibodies, and can detect proteins barcoded into multiple antibodies and more than 20,000 genes $[63,64]$.

Several techniques unaffected by cellular heterogeneity have been developed to overcome the limitations of single transcriptome studies. Single-cell multiomics techniques can distinguish subtypes of cells from heterogeneous cell populations [65-67]. In addition, the accuracy of lineage trajectory analysis can be enhanced by integrating gene expression and the epigenetic changes that occur during cell division and delivery to daughter cells [68]. Single-cell multiomics data can reveal correlations between different -omics data, and presumably, may effectively reveal the causal relationship between -omics and technology development.

\section{APPROACHES AND UTILITY OF SINGLE-CELL TRANSCRIPTOMICS IN NEPHROLOGY}

\section{Kidney single-cell atlas}

Several scRNA-seq studies of human and mouse diseased and normal kidneys have been conducted; the results were applied to produce a variety of databases (Table 1).

The creation of a single-cell atlas offers several advantages. First, by typing differentiated cells, their stage of development and differentiation can be determined. Second, it provides insight into the mechanisms of disease development and progression. By analyzing diseased tissue at the single-cell level and comparing it with the cell atlas of healthy tissue, it is possible to evaluate the heterogeneity of cells in the diseased tissue and so determine the cause of the disease. Analyzing single cells in diseased tissues can identify the factors that contribute to differences between individuals, allowing personalized treatment and facilitating the development of new drugs and biomarkers.

Park et al. [6] constructed a cell atlas using scRNA-seq data of 57,979 cells from healthy mouse kidneys. They identified 18 kidney epithelial and immune cell types as well as novel transitional cell types located between intercalated cells (ICs) and principal cells (PCs) in the collecting ducts. Moreover, they revealed that Notch signaling is responsible for the transition of ICs to PCs. Karaiskos et al. [69] performed single-cell profiling of mouse glomerulus cells. They identified glomerular cell types such as podocytes, mesangial cells, and endothelial cells. They also revealed novel marker genes for all glomerular cell types and assessed the transcriptional heterogeneity of each cell type via subclustering the endothelial cells and podocytes. Chen et al. [70] performed scRNA-seq on mouse collecting duct cells. Ransick et al. [71] anatomically dissected male and female kidneys for anatomy-guided scRNA-seq. They confirmed sexual, spatial, and temporal diversity in nephrons and the collecting system.

Liao et al. [72] identified 10 normal human cell clusters via scRNA-seq of 23,366 kidney cells from three human donors. Proximal tubule cells and collecting duct cells were classified into three and two subtypes, respectively. For gene expression profiling of human fetal kidney development, Wang et al. [73] applied scRNA-seq to 3,543 renal cells spanning several embryonic stages and classified the major cell types. Moreover, they identified two subpopulations in the cap mesenchyme and assessed the molecular heterogeneity of the cap mesenchyme. Furthermore, they identified the transcription factors and signaling pathways involved in nephron tubule segmentation during fetal kidney development. Wu et al. [74] performed scRNA-seq of human kidney allograft biopsy samples and confirmed the proinflammatory response of allograft rejection by comparison with healthy kidney epithelial transcriptomes. Understanding the characteristics and origins of organ-specific tumors is crucial for making treatment decisions. Young et al. [75] performed single-cell profiling of human renal tumors and normal tissue from pediatric and adult kidneys. They confirmed that Wilms tumor, a pediatric kidney cancer, was derived from abnormal fetal cells. The origin of the tumor was predicted by matching the transcriptome of adult kidney cancer to a specific subtype of proximal convoluted tubular cells. Lake et al. [7] optimized the snRNA-seq pipeline for clinical specimens to define the molecular transition states of more than 
Table 1. Single-cell analysis databases

\begin{tabular}{|c|c|c|c|c|c|}
\hline Definition & Resource & Target disease & Species & $\begin{array}{c}\text { Cell } \\
\text { number }\end{array}$ & Platform \\
\hline $\begin{array}{l}\text { Single-cell atlas from healthy } \\
\text { mouse kidneys [6] }\end{array}$ & http://susztaklab.com/sc & Normal & Mouse & 57,979 & 10x Genomics \\
\hline $\begin{array}{l}\text { Single-cell atlas of the mouse } \\
\text { glomeruli [69] }\end{array}$ & $\begin{array}{l}\text { https://shiny.mdc-berlin.de/ } \\
\text { mgsca/ }\end{array}$ & Normal & Mouse & $\sim 13,000$ & Drop-seq \\
\hline $\begin{array}{l}\text { Single-cell RNA sequencing for } \\
\text { each collecting duct type [70] }\end{array}$ & $\begin{array}{l}\text { https://hpcwebapps.cit.nih. } \\
\text { gov/ESBL/Database/scRNA- } \\
\text { Seq/alpha-fraction.html }\end{array}$ & Normal & Mouse & 235 & Fluidigm C1 \\
\hline $\begin{array}{l}\text { Profiling kidney cell by lineage, } \\
\text { gender, zonation [71] }\end{array}$ & $\begin{array}{l}\text { https://cello.shinyapps.io/ } \\
\text { kidneycellexplorer/ }\end{array}$ & Normal & Mouse & 31,265 & 10x Genomics \\
\hline $\begin{array}{l}\text { Single-cell RNA sequencing of } \\
\text { human kidney [72] }\end{array}$ & & Normal & Human & 23,366 & 10x Genomics \\
\hline $\begin{array}{l}\text { Profiling of human kidney } \\
\text { development by single-cell RNA } \\
\text { sequencing [73] }\end{array}$ & & $\begin{array}{c}\text { Kidney } \\
\text { development }\end{array}$ & Human & $\sim 3,000$ & STRT-seq \\
\hline $\begin{array}{l}\text { Inflammatory response in } \\
\text { transplantation kidney [74] }\end{array}$ & $\begin{array}{l}\text { http://humphreyslab.com/ } \\
\text { SingleCell/ }\end{array}$ & Transplantation & Human & 4,487 & InDrops \\
\hline $\begin{array}{l}\text { Single nucleus RNA sequencing of } \\
\text { human kidney [7] }\end{array}$ & & Normal & Human & $\begin{array}{l}17,659 \\
\text { (nuclei) }\end{array}$ & Drop-seq \\
\hline $\begin{array}{l}\text { Single-cell atlas of the human } \\
\text { kidney's immune system from } \\
\text { mature and fetal kidneys [76] }\end{array}$ & $\begin{array}{l}\text { https://www.kidneycellatlas. } \\
\text { org/ }\end{array}$ & Normal & Human & 67,471 & 10x Genomics \\
\hline $\begin{array}{l}\text { Human kidney cell atlas by Mux- } \\
\text { seq [77] }\end{array}$ & & Normal & Human & 45,000 & 10x Genomics \\
\hline $\begin{array}{l}\text { Single-cell RNA sequencing from } \\
\text { patients with lupus nephritis [78] }\end{array}$ & & Lupus nephritis & Human & 4,019 & Fluidigm $\mathrm{C}_{1}$ \\
\hline $\begin{array}{l}\text { Kidney immune cell profiling by } \\
\text { single-cell RNA sequencing in } \\
\text { patients with lupus nephritis [79] }\end{array}$ & $\begin{array}{l}\text { https://immunogenomics.io/ } \\
\text { ampsle/ }\end{array}$ & Lupus nephritis & Human & 8,455 & CEL-seq2 \\
\hline $\begin{array}{l}\text { Single-cell atlas of the human } \\
\text { diabetes kidney [8o] }\end{array}$ & $\begin{array}{l}\text { http://humphreyslab.com/ } \\
\text { SingleCell/ }\end{array}$ & Diabetes & Human & $\begin{array}{l}23,980 \\
\text { (nuclei) }\end{array}$ & 10x Genomics \\
\hline $\begin{array}{l}\text { Single-cell RNA sequencing of } \\
\text { mouse glomeruli with diabetes [81] }\end{array}$ & & Diabetes & Mouse & 644 & Fluidigm C1 \\
\hline $\begin{array}{l}\text { Single-cell RNA sequencing from } \\
\text { UUO mouse model [82] }\end{array}$ & $\begin{array}{l}\text { https://argonaut.is.ed.ac.uk/ } \\
\text { shiny/katie.connor/mac_- } \\
\text { shiny/ }\end{array}$ & UUO & Mouse & 25,381 & $\begin{array}{l}\text { 10x } \\
\text { Genomics, } \\
\text { SMART-seq2 }\end{array}$ \\
\hline
\end{tabular}

UUO, unilateral ureteric obstruction.

10 nephron segments in the proximal tubules and collecting ducts. Using the results they described the anatomical nephron organization, providing a starting point for building a molecular and physiological atlas that can be used to identify variations in kidney diseases. Stewart et al. [76] evaluated the spatiotemporal immune topology in human kidneys by scRNA-seq. scRNA-seq of mature and fetal kidneys revealed the asymmetric distribution of immune cells, and the contribution of each cell type based on the nephrogenesis stage was analyzed. This kidney immune cell atlas provides insight into the pathogenetic mechanisms and therapeutic targets in immune and infectious kidney diseases.

Single-cell studies of human and mouse kidneys have 
shown the complexity of kidney tissues; however, some scRNA-seq studies missed known cell subtypes. This is because sample preparation, dissociation, and batch effects influence the detection of rare or sensitive cells. A multiplex approach to droplet snRNA-seq (Mux-seq) was applied to minimize batch variation [77]. This allows scRNA-seq of several human biopsies simultaneously, enabling identification of kidney cell populations in health and disease.

\section{Single-cell analysis of kidney diseases}

scRNA-seq of the kidneys of patients with lupus nephritis (LN) has been reported. Der et al. [78] reported that the type 1 interferon response in tubular epithelial cells was greater than that in healthy controls, and confirmed clinically relevant disease signatures in kidney and skin biopsies. Arazi et al. [79] performed scRNA-seq of the kidney tissues of patients with LN and healthy controls, and found that leukocytes in the kidney were active and that their activation status differed before and after the inflammatory reaction. Subgroups of innate and adaptive immune cells expressing various transcription factors related to systemic lupus erythematous were identified by analyzing cluster-specific expression of risk-related genes in genome-wide association studies. scRNA-seq of LN tissues confirmed the molecular signature associated with prognosis, enabling improvement of patient care and stratification.

Transcriptome profiling of kidney tissue or isolated glomeruli provides insight into the pathogenesis of kidney fibrosis. Wilson et al. [8o] performed snRNA-seq using 23,980 nuclei from control and diabetic kidney cortex samples, and found gene expression changes in diabetic glomeruli, mesangial cells, endothelial cells, and diabetic proximal convoluted tubules and ascending limbs. In addition, differential expression of ligand-receptors was observed in the various glomerular cell types. Differential expression analysis of leukocytes revealed that infiltrating immune cells contribute to the synthesis of kidney risk inflammatory signature markers. Such gene expression changes may enable identification of biomarkers and signaling pathways early in diabetic nephropathy. To evaluate the mechanism of early diabetic kidney disease, Fu et al. [81] performed scRNAseq of kidney glomerular cells in a diabetic mouse model. They identified five distinct cell clusters and novel glomerular cell-specific markers. Comparison of the scRNA-seq data between diabetic and normal mouse kidneys revealed identical cell clusters, but the immune cell population was increased in diabetic mice. Moreover, there were remarkable gene expression changes in endothelial and mesangial cells in the diabetic mouse kidney. These analyses identified key factors involved in diabetic kidney disease progression and thereby assisted development of new treatment approaches [81]. Using the reversible unilateral ureteric obstruction model (R-UUO), changes in renal injury and repair at the single-cell level were noted [82]. scRNA-seq revealed myeloid cell heterogeneity in damaged and recovering kidneys by identifying new monocyte and macrophage subsets not observed in the kidney [82]. The data will enable identification of potential therapeutic targets and the development of therapeutics for kidney diseases.

Intratumoral heterogeneity interferes with marker-based cancer treatment because targeted therapy removes only a specific population of tumor cells. To evaluate the molecular and cellular heterogeneity in renal cell carcinoma, Kim et al. [83] performed transcriptome profiling of primary and metastatic renal cell carcinoma with single-cell resolution. Metastatic cancer cells exhibited distinct gene expression patterns with increased metastatic and aggressive signatures compared to primary cancer cells. Based on transcriptome profiling and drug screening, the drug sensitivity and activation status of signaling pathways were predicted, and the correlation between the predicted signature and measured data was verified. sci-RNA-seq allows identification of cell subsets with active signaling pathways and selection of the most effective drug combination. Such approaches will overcome the intratumoral heterogeneity that hampers precision medicine. The immune cells of the tumor microenvironment (TME) are crucial in determining the response to cancer immunotherapy. Nevertheless, the role of immune cells in clear cell renal cell carcinoma (ccRCC) is unclear, and most patients do not respond to these treatments. Vishwakarma et al. [84] applied scRNA-seq to the tumors and peripheral blood immune cells from patients with ccRCC to characterize the TME. They identified several intratumoral CD8 T cell states that characterize the effector, memory, and exhausted subpopulations together with multiple states of tumor-associated macrophages and dendritic cells. 
Moreover, they found intratumoral cytotoxic and regulatory $\mathrm{CD}_{4} \mathrm{~T}$ cell clusters, which led to the establishment of tumor-infiltrating effectors and memory cells. These results will facilitate research on TME to identify new therapeutic targets and biomarkers.

\section{APPLICATION OF SINGLE-CELL ANALYSIS IN PRECISION MEDICINE}

Single-cell analysis accelerates research on diseases related to genetic or epigenetic alterations. Single-cell analysis has been applied in microbiology, immunology, neurology, and oncology and has the potential to improve diagnostics, prognostics, targeted therapy, early detection, and noninvasive monitoring [85].

Existing drug screening methods provide only rough readouts such as cell survival, proliferation, altered cell morphology, or specific molecular findings revealing whether a specific enzyme is blocked. Therefore, most assays miss the cell-state changes or subtle changes in gene expression that can reveal intracellular mechanisms. In addition, it may not be possible to detect unexpected side effects of a drug or different responses of genetically identical cells. During drug development, single-cell sequencing provides detailed information based on the genetic, epigenetic, and transcriptomic profiles of responders compared to nonresponders, and can improve efficiency [86]. Srivatsan et al. [87] introduced sci-Plex (single-cell combinatorial indexing RNAseq for multiplex transcriptomics), which combines nuclear hashing and conventional sci-RNA-seq to quantify the global transcriptional responses to independent perturbations with single-cell resolution. Sci-Plex can distinguish the effect of a drug on a cellular subset and can reveal the heterogeneity of the cellular response to perturbation. Furthermore, it is possible to measure changes in the relative proportions of a subset of distinct cells. Shin et al. [40] developed a multiplexed scRNA-seq method for profiling multiple experimental conditions, and evaluated its ability to perform single-cell transcriptome profiling for multiple drugs. The results showed that each drug had a unique transcriptome response and gene expression signature at the single-cell level. The method can be applied to screening drugs and their transcriptional responses in a high-throughput man- ner. Kim et al. [83] performed drug screening for renal cell carcinoma based on sci-RNA-seq. They identified cellular subpopulations with active signaling pathways and combinations of drugs that eliminate cancer cells [83]. This allows assessment of the heterogeneous cellular pattern of diseases, facilitating drug screening.

Immunotherapy shows promise for treating diseases at advanced stages. Much progress has been made in the development of cancer immunotherapies. Single-cell sequencing will help in understanding tumor heterogeneity and highlight the need for this technique to develop safe and effective treatments. For instance, Krieg et al. [88] analyzed immune cell subsets at the single-cell level in the peripheral blood of patients before and after treatment to investigate the immune signature related to the response to anti-programmed cell death protein-1 (anti-PD-1) immunotherapy. Using scRNA-seq, Kim et al. [89] reported that the concentration of exhausting induction factors that cause $\mathrm{T}$ cells to lose their potency against abnormal cells can predict patient-specific reactions to cancer immunotherapy. Chimeric antigen receptors (CAR) are genetically modified to give $\mathrm{T}$ cells the ability to target specific proteins. CAR T cell therapy uses modified $\mathrm{T}$ cells to target and destroy cancer cells. Suarez et al. [90] designed a new CAR therapy targeting carbonic anhydrase (CAIX) to prevent T cells exhaustion. Anti-CAIX-CAR T cells secreting anti-PD-L1 reduced $T$ cells exhaustion and improved the efficacy of CAR T cells treatment for ccRCC in vivo. Sheih et al. [91] performed scRNA-seq on CD8+ CAR T cells from patients receiving CAR $\mathrm{T}$ cells immunotherapy and from the infusion product. scRNA-seq can provide insight into the in vivo behavior of CAR T cells after adoptive transfer and may enhance CAR T cells immunotherapy.

Single-cell analysis is enabling precision medicine in oncology; however, precision medicine in nephrology lags that in other fields. Insight into immune mechanisms is critical for developing precision medicine for kidney disease [92]. To identify the key elements, the immune cell heterogeneity of tissue samples can be resolved by single-cell analysis. A previous study assessed the human kidney spatiotemporal immune topology at the single-cell level [76], providing a description of the renal immune system and facilitating diagnosis and treatment of kidney diseases. Furthermore, single-cell multiomics analysis enables evaluation of disease-caus- 
ing factors and their interactions, overcoming the limitations of single-omics data. Therefore, single-cell analysis will contribute to the development of precision medicine in nephrology (Fig. 1).

\section{CONCLUSIONS}

Precision medicine is a new medical approach that takes into account genetic variability, environment, and lifestyle. Based on specific genetic biomarkers and -omics approaches, precision medicine has had a remarkable influence on, for instance, renal cancer and acute and chronic kidney diseases. Precision medicine differs from the one-size-fits-all approach, which does not consider individual differences. Identifying specific target genes, critical signaling pathways, and cell types is the first step in the transition from conventional to precision medicine in nephrology. In contrast to identifying target genes, little effort has focused on identifying cellular targets in kidney disease because of technical limitations. Moreover, bulk approaches cannot differentiate primary cell autonomous responses, which lead to disease development, from secondary cell non-autonomous responses. scRNA-seq is an innovative approach and plays a pivotal role in precision medicine by enabling characterization of the cells, pathways, and genes involved in disease pathogenesis.

sci-RNA-seq can reveal new biological processes by combining multiple datasets, allowing a detailed description of the state of a single-cell. Multiomics approaches have been used to characterize disease states and identify therapeutic targets or biomarkers for kidney diseases. A variety of single-cell sequencing technologies is capable of analyzing the genome, epigenome, proteome, and transcriptome of a single-cell. Single-cell sequencing technologies enable simultaneous genomic, epigenomic, transcriptomic, and proteomic analyses of the same cell.

Integrative single-cell transcriptome data will promote untangling of the complex pathological mechanisms of kidney diseases and allow assessment of disease risk and monitoring of disease status, thus enabling personalized medicine.

\section{Conflict of interest}

No potential conflict of interest relevant to this article was reported.

\section{Acknowledgments}

This work was supported by "Global University Project (GUP)," "GIST Research Institute (GRI)," and "GIST Research Institute (GRI) IIBR" grants funded by the GIST in $202 \mathrm{O}$.

\section{REFERENCES}

1. Wyatt CM, Schlondorff D. Precision medicine comes of age in nephrology: identification of novel biomarkers and therapeutic targets for chronic kidney disease. Kidney Int 2016;89:734-737.

2. Denisenko E, Guo BB, Jones M, et al. Systematic assessment of tissue dissociation and storage biases in single-cell and single-nucleus RNA-seq workflows. Genome Biol 2020;21:130.

3. Nguyen QH, Pervolarakis N, Nee K, Kessenbrock K. Experimental considerations for single-cell RNA sequencing approaches. Front Cell Dev Biol 2018;6:108.

4. Wolock SL, Lopez R, Klein AM. Scrublet: computational identification of cell doublets in single-cell transcriptomic data. Cell Syst 2019;8:281-291.

5. McGinnis CS, Murrow LM, Gartner ZJ. DoubletFinder: doublet detection in single-cell RNA sequencing data using artificial nearest neighbors. Cell Syst 2019;8:329-337.

6. Park J, Shrestha R, Qiu C, et al. Single-cell transcriptomics of the mouse kidney reveals potential cellular targets of kidney disease. Science 2018;360:758-763.

7. Lake BB, Chen S, Hoshi M, et al. A single-nucleus RNA-sequencing pipeline to decipher the molecular anatomy and pathophysiology of human kidneys. Nat Commun 2019;10:2832.

8. Wu H, Kirita Y, Donnelly EL, Humphreys BD. Advantages of single-nucleus over single-cell RNA sequencing of adult kidney: rare cell types and novel cell states revealed in fibrosis. J Am Soc Nephrol 2019;30:23-32.

9. Ziegenhain C, Vieth B, Parekh S, et al. Comparative analysis of single-cell RNA sequencing methods. Mol Cell 2017;65:631-643.

10. Deng Q, Ramskold D, Reinius B, Sandberg R. Single-cell RNA-seq reveals dynamic, random monoallelic gene ex- 
pression in mammalian cells. Science 2014;343:193-196.

11. Reinius B, Mold JE, Ramskold D, et al. Analysis of allelic expression patterns in clonal somatic cells by single-cell RNA-seq. Nat Genet 2016;48:1430-1435.

12. Tseng E, Underwood JG. Single-cell full-length isoform characterization using SMRT sequencing: Pacific Biosciences' Iso-Seq method produces highly accurate long reads that can be used in combination with shortread RNA-seq approaches. Genet Eng Biotechnol News 2020;40:58-60.

13. Song Y, Xu X, Wang W, Tian T, Zhu Z, Yang C. Single cell transcriptomics: moving towards multi-omics. Analyst 2019;144:3172-3189.

14. Ramskold D, Luo S, Wang YC, et al. Full-length mRNA-Seq from single-cell levels of RNA and individual circulating tumor cells. Nat Biotechnol 2012;30:777-782.

15. Hayashi T, Ozaki H, Sasagawa Y, Umeda M, Danno H, Nikaido I. Single-cell full-length total RNA sequencing uncovers dynamics of recursive splicing and enhancer RNAs. Nat Commun 2018;9:619.

16. Picelli S, Bjorklund AK, Faridani OR, Sagasser S, Winberg G, Sandberg R. Smart-seq2 for sensitive full-length transcriptome profiling in single cells. Nat Methods 2013;10:1096-1098.

17. Sasagawa Y, Nikaido I, Hayashi T, et al. Quartz-Seq: a highly reproducible and sensitive single-cell RNA sequencing method, reveals non-genetic gene-expression heterogeneity. Genome Biol 2013;14:R31.

18. Hashimshony T, Senderovich N, Avital G, et al. CEL-Seq2: sensitive highly-multiplexed single-cell RNA-Seq. Genome Biol 2016;17:77.

19. Macosko EZ, Basu A, Satija R, et al. Highly parallel genome-wide expression profiling of individual cells using nanoliter droplets. Cell 2015;161:1202-1214.

20. Klein AM, Mazutis L, Akartuna I, et al. Droplet barcoding for single-cell transcriptomics applied to embryonic stem cells. Cell 2015;161:1187-1201.

21. Zheng GX, Terry JM, Belgrader P, et al. Massively parallel digital transcriptional profiling of single cells. Nat Commun 2017;8:14049.

22. Han X, Wang R, Zhou Y, et al. Mapping the mouse cell atlas by Microwell-Seq. Cell 2018;172:1091-1107.

23. Gierahn TM, Wadsworth MH 2nd, Hughes TK, et al. SeqWell: portable, low-cost RNA sequencing of single cells at high throughput. Nat Methods 2017;14:395-398.

24. Cao J, Packer JS, Ramani V, et al. Comprehensive sin- gle-cell transcriptional profiling of a multicellular organism. Science 2017;357:661-667.

25. Rosenberg AB, Roco CM, Muscat RA, et al. Single-cell profiling of the developing mouse brain and spinal cord with split-pool barcoding. Science 2018;360:176-182.

26. Femino AM, Fay FS, Fogarty K, Singer RH. Visualization of single RNA transcripts in situ. Science 1998;280:585590.

27. Raj A, van den Bogaard P, Rifkin SA, van Oudenaarden A, Tyagi S. Imaging individual mRNA molecules using multiple singly labeled probes. Nat Methods 2008;5:877-879.

28. Eng CL, Lawson $\mathrm{M}$, Zhu Q, et al. Transcriptome-scale super-resolved imaging in tissues by RNA seqFISH. Nature 2019;568:235-239.

29. Wang G, Moffitt JR, Zhuang X. Multiplexed imaging of high-density libraries of RNAs with MERFISH and expansion microscopy. Sci Rep 2018;8:4847.

30. Wang X, Allen WE, Wright MA, et al. Three-dimensional intact-tissue sequencing of single-cell transcriptional states. Science 2018;361:eaat5691.

31. Lee JH, Daugharthy ER, Scheiman J, et al. Fluorescent in situ sequencing (FISSEQ) of RNA for gene expression profiling in intact cells and tissues. Nat Protoc 2015;10:442-458.

32. Stahl PL, Salmen F, Vickovic S, et al. Visualization and analysis of gene expression in tissue sections by spatial transcriptomics. Science 2016;353:78-82.

33. Nitzan M, Karaiskos N, Friedman N, Rajewsky N. Gene expression cartography. Nature 2019;576:132-137.

34. Karaiskos N, Wahle P, Alles J, et al. The drosophila embryo at single-cell transcriptome resolution. Science 2017;358:194-199.

35. Satija R, Farrell JA, Gennert D, Schier AF, Regev A. Spatial reconstruction of single-cell gene expression data. Nat Biotechnol 2015;33:495-502.

36. van den Brink SC, Sage F, Vertesy A, et al. Single-cell sequencing reveals dissociation-induced gene expression in tissue subpopulations. Nat Methods 2017;14:935-936.

37. Chen W, Zhang S, Williams J, et al. A comparison of methods accounting for batch effects in differential expression analysis of UMI count based single cell RNA sequencing. Comput Struct Biotechnol J 2020;18:861-873.

38. Tran HTN, Ang KS, Chevrier M, et al. A benchmark of batch-effect correction methods for single-cell RNA sequencing data. Genome Biol 2020;21:12.

39. Stoeckius M, Zheng S, Houck-Loomis B, et al. Cell hash- 
ing with barcoded antibodies enables multiplexing and doublet detection for single cell genomics. Genome Biol 2018;19:224.

40. Shin D, Lee W, Lee JH, Bang D. Multiplexed single-cell RNA-seq via transient barcoding for simultaneous expression profiling of various drug perturbations. Sci Adv 2019;5:eaav2249.

41. Zilionis R, Nainys J, Veres A, et al. Single-cell barcoding and sequencing using droplet microfluidics. Nat Protoc 2017;12:44-73.

42. Azizi E, Carr AJ, Plitas G, et al. Single-cell map of diverse immune phenotypes in the breast tumor microenvironment. Cell 2018;174:1293-1308.

43. Parekh S, Ziegenhain C, Vieth B, Enard W, Hellmann I. zUMIs: a fast and flexible pipeline to process RNA sequencing data with UMIs. Gigascience 2018;7:giyo59.

44. AlJanahi AA, Danielsen M, Dunbar CE. An introduction to the analysis of single-cell RNA-sequencing data. Mol Ther Methods Clin Dev 2018;10:189-196.

45. Luecken MD, Theis FJ. Current best practices in single-cell RNA-seq analysis: a tutorial. Mol Syst Biol 2019;15:e8746.

46. McCarthy DJ, Campbell KR, Lun AT, Wills QF. Scater: pre-processing, quality control, normalization and visualization of single-cell RNA-seq data in R. Bioinformatics 2017;33:1179-1186.

47. Hwang B, Lee JH, Bang D. Single-cell RNA sequencing technologies and bioinformatics pipelines. Exp Mol Med 2018;50:96.

48. Lun AT, Bach K, Marioni JC. Pooling across cells to normalize single-cell RNA sequencing data with many zero counts. Genome Biol 2016;17:75.

49. Peyvandipour A, Shafi A, Saberian N, Draghici S. Identification of cell types from single cell data using stable clustering. Sci Rep 2020;10:12349.

50. Townes FW, Hicks SC, Aryee MJ, Irizarry RA. Feature selection and dimension reduction for single-cell RNA-Seq based on a multinomial model. Genome Biol 2019;20:295.

51. van der Maaten L, Hinton G. Visualizing data using t-SNE. J Mach Learn Res 2008;9:2579-2605.

52. Becht E, McInnes L, Healy J, et al. Dimensionality reduction for visualizing single-cell data using UMAP. Nat Biotechnol 2019;37:38-44.

53. Stachelscheid H, Seltmann S, Lekschas F, et al. CellFinder: a cell data repository. Nucleic Acids Res 2014;42(Database issue):D950-D958.

54. Zhang X, Lan Y, Xu J, et al. CellMarker: a manually curated resource of cell markers in human and mouse. Nucleic Acids Res 2019;47:D721-D728.

55. Franzen O, Gan LM, Bjorkegren JLM. PanglaoDB: a web server for exploration of mouse and human single-cell RNA sequencing data. Database (Oxford) 2019;2019:bazo46.

56. Abdelaal T, Michielsen L, Cats D, et al. A comparison of automatic cell identification methods for single-cell RNA sequencing data. Genome Biol 2019;20:194.

57. Lafzi A, Moutinho C, Picelli S, Heyn H. Tutorial: guidelines for the experimental design of single-cell RNA sequencing studies. Nat Protoc 2018;13:2742-2757.

58. Macaulay IC, Ponting CP, Voet T. Single-cell multiomics: multiple measurements from single cells. Trends Genet 2017;33:155-168.

59. Macaulay IC, Haerty W, Kumar P, et al. G\&T-seq: parallel sequencing of single-cell genomes and transcriptomes. Nat Methods 2015;12:519-522.

6o. Angermueller C, Clark SJ, Lee HJ, et al. Parallel single-cell sequencing links transcriptional and epigenetic heterogeneity. Nat Methods 2016;13:229-232.

61. Hu Y, Huang K, An Q, et al. Simultaneous profiling of transcriptome and DNA methylome from a single cell. Genome Biol 2016;17:88.

62. Cao J, Cusanovich DA, Ramani V, et al. Joint profiling of chromatin accessibility and gene expression in thousands of single cells. Science 2018;361:1380-1385.

63. Peterson VM, Zhang KX, Kumar N, et al. Multiplexed quantification of proteins and transcripts in single cells. Nat Biotechnol 2017;35:936-939.

64. Stoeckius M, Hafemeister C, Stephenson W, et al. Simultaneous epitope and transcriptome measurement in single cells. Nat Methods 2017;14:865-868.

65. Luo C, Keown CL, Kurihara L, et al. Single-cell methylomes identify neuronal subtypes and regulatory elements in mammalian cortex. Science 2017;357:600-604.

66. Tang X, Huang Y, Lei J, Luo H, Zhu X. The single-cell sequencing: new developments and medical applications. Cell Biosci 2019;9:53.

67. Ortega MA, Poirion O, Zhu X, et al. Using single-cell multiple omics approaches to resolve tumor heterogeneity. Clin Transl Med 2017;6:46.

68. Hu Y, An Q, Sheu K, Trejo B, Fan S, Guo Y. Single cell multi-omics technology: methodology and application. Front Cell Dev Biol 2018;6:28.

69. Karaiskos N, Rahmatollahi M, Boltengagen A, et al. A sin- 
gle-cell transcriptome atlas of the mouse glomerulus. J Am Soc Nephrol 2018;29:2060-2068.

70. Chen L, Lee JW, Chou CL, et al. Transcriptomes of major renal collecting duct cell types in mouse identified by single-cell RNA-seq. Proc Natl Acad Sci U S A 2017;114:E9989-E9998.

71. Ransick A, Lindstrom NO, Liu J, et al. Single-cell profiling reveals sex, lineage, and regional diversity in the mouse kidney. Dev Cell 2019;51:399-413.

72. Liao J, Yu Z, Chen Y, et al. Single-cell RNA sequencing of human kidney. Sci Data 2020;7:4.

73. Wang P, Chen Y, Yong J, et al. Dissecting the global dynamic molecular profiles of human fetal kidney development by single-cell RNA sequencing. Cell Rep 2018;24:3554-3567.

74. Wu H, Malone AF, Donnelly EL, et al. Single-cell transcriptomics of a human kidney allograft biopsy specimen defines a diverse inflammatory response. J Am Soc Nephrol 2018;29:2069-2080.

75. Young MD, Mitchell TJ, Vieira Braga FA, et al. Single-cell transcriptomes from human kidneys reveal the cellular identity of renal tumors. Science 2018;361:594-599.

76. Stewart BJ, Ferdinand JR, Young MD, et al. Spatiotemporal immune zonation of the human kidney. Science 2019;365:1461-1466.

77. Schroeder AW, Sur S, Rashmi P, et al. Novel human kidney cell subsets identified by Mux-Seq. Transplantation 2020;104(Suppl 3):S85.

78. Der E, Suryawanshi H, Morozov P, et al. Tubular cell and keratinocyte single-cell transcriptomics applied to lupus nephritis reveal type I IFN and fibrosis relevant pathways. Nat Immunol 2019;20:915-927.

79. Arazi A, Rao DA, Berthier CC, et al. The immune cell landscape in kidneys of patients with lupus nephritis. Nat Immunol 2019;20:902-914.

8o. Wilson PC, Wu H, Kirita Y, et al. The single-cell transcriptomic landscape of early human diabetic nephropathy. Proc Natl Acad Sci U S A 2019;116:19619-19625.

81. Fu J, Akat KM, Sun Z, et al. Single-cell RNA profiling of glomerular cells shows dynamic changes in experimental diabetic kidney disease. J Am Soc Nephrol 2019;30:533-545.

82. Conway BR, O'Sullivan ED, Cairns C, et al. Kidney single-cell atlas reveals myeloid heterogeneity in progression and regression of kidney disease. J Am Soc Nephrol 2020;31:2833-2854.

83. Kim KT, Lee HW, Lee HO, et al. Application of single-cell RNA sequencing in optimizing a combinatorial therapeutic strategy in metastatic renal cell carcinoma. Genome Biol 2016;17:80.

84. Vishwakarma A, Bocherding N, Chimenti MS, et al. Mapping the immune landscape of clear cell renal cell carcinoma by single-cell RNA-seq. bioRxiv 2019 Oct 31. https:// doi.org/10.1101/824482.

85. Wiedmeier JE, Noel P, Lin W, Von Hoff DD, Han H. Single-cell sequencing in precision medicine. Cancer Treat Res 2019;178:237-252.

86. Xu X, Hou Y, Yin X, et al. Single-cell exome sequencing reveals single-nucleotide mutation characteristics of a kidney tumor. Cell 2012;148:886-895.

87. Srivatsan SR, McFaline-Figueroa JL, Ramani V, et al. Massively multiplex chemical transcriptomics at single-cell resolution. Science 2020;367:45-51.

88. Krieg C, Nowicka M, Guglietta S, et al. High-dimensional single-cell analysis predicts response to anti-PD-1 immunotherapy. Nat Med 2018;24:144-153.

89. Kim K, Park S, Park SY, et al. Single-cell transcriptome analysis reveals TOX as a promoting factor for T cell exhaustion and a predictor for anti-PD-1 responses in human cancer. Genome Med 2020;12:22.

90. Suarez ER, Chang de K, Sun J, et al. Chimeric antigen receptor $\mathrm{T}$ cells secreting anti-PD-L1 antibodies more effectively regress renal cell carcinoma in a humanized mouse model. Oncotarget 2016;7:34341-34355.

91. Sheih A, Voillet V, Hanafi LA, et al. Clonal kinetics and single-cell transcriptional profiling of CAR-T cells in patients undergoing CD19 CAR-T immunotherapy. Nat Commun 2020;11:219.

92. Kurts C, Panzer U, Anders HJ, Rees AJ. The immune system and kidney disease: basic concepts and clinical implications. Nat Rev Immunol 2013;13:738-753. 